\title{
Existence Result of Generalized Vector Quasiequilibrium Problems in Locally G-Convex Spaces
}

\author{
Somyot Plubtieng and Kanokwan Sitthithakerngkiet \\ Department of Mathematics, Faculty of Science, Naresuan University, Phitsanulok 65000, Thailand \\ Correspondence should be addressed to Somyot Plubtieng, somyotp@nu.ac.th \\ Received 30 November 2010; Accepted 18 February 2011 \\ Academic Editor: Yeol J. Cho
}

Copyright (C) 2011 S. Plubtieng and K. Sitthithakerngkiet. This is an open access article distributed under the Creative Commons Attribution License, which permits unrestricted use, distribution, and reproduction in any medium, provided the original work is properly cited.

This paper deals with the generalized strong vector quasiequilibrium problems without convexity in locally G-convex spaces. Using the Kakutani-Fan-Glicksberg fixed point theorem for upper semicontinuous set-valued mapping with nonempty closed acyclic values, the existence theorems for them are established. Moreover, we also discuss the closedness of strong solution set for the generalized strong vector quasiequilibrium problems.

\section{Introduction}

Let $X$ be real topological vector space, and let $C$ be a nonempty closed convex subset of $X$. Let $F: C \times C \rightarrow \mathbb{R}$ be a bifunction, where $\mathbb{R}$ is the set of real numbers. The equilibrium problem for $F$ is to find $x \in C$ such that

$$
F(x, y) \geq 0 \quad \forall y \in C
$$

Problem (1.1) was studied by Blum and Oettli [1]. The set of solution of (1.1) is denoted by $\operatorname{EP}(F)$. The equilibrium problem contains many important problems as special cases, including optimization, Nash equilibrium, complementarity, and fixed point problems (see [1-3] and the references therein). Recently, there has been an increasing interest in the study of vector equilibrium problems. Many results on the existence of solutions for vector variational inequalities and vector equilibrium problems have been established (see, e.g., [416]). 
Let $X$ and $Y$ be real topological vector spaces and $K$ a nonempty subset of $X$. Let $C$ be a closed and convex cone in $Y$ with int $C(x) \neq \emptyset$, where int $C(x)$ denotes the topological interior of $C$. For a bifunction $F: K \times K \rightarrow Y$, the vector equilibrium problem (for short, $\mathrm{VEP}$ ) is to find $x \in K$ such that

$$
F(x, y) \notin-\operatorname{int} C, \quad \forall y \in K,
$$

which is a unified model of several known problems, for instance, vector variational and variational-like inequality problems, vector complementarity problem, vector optimization problem, and vector saddle point problem; see, for example, $[3,8,17,18]$ and references therein. In 2003, Ansari and Yao [19] introduced vector quasiequilibrium problem (for short, VQEP) to find $x \in K$ such that

$$
x \in A(x): F(x, y) \notin-\operatorname{int} C \quad \forall y \in A(x),
$$

where $A: K \rightarrow 2^{K}$ is a multivalued map with nonempty values.

Recently, Ansari et al. [4] considered a more general problem which contains VEP and generalized vector variational inequality problems as special cases. Let $X$ and $Z$ be real locally convex Hausdorff space, $K \subset X$ a nonempty subset and $C \subset Z$ a closed convex pointed cone. Let $F: K \times K \rightarrow 2^{Z}$ be a given set-valued mapping. Ansari et al. [4] introduced the following problems, to find $x \in K$ such that

$$
F(x, y) \not \subset-\operatorname{int} C \quad \forall y \in K,
$$

or to find $x \in K$ such that

$$
F(x, y) \subset C \quad \forall y \in K
$$

It is called generalized vector equilibrium problem (for short, GVEP), and it has been studied by many authors; see, for example, [20-22] and references therein. For other possible ways to generalize VEP, we refer to [23-25]. If int $C$ is nonempty and $x$ satisfies (1.4), then we call $x$ a weak efficient solution for VEP, and if $x$ satisfies (1.5), then we call $x$ a strong solution for VEP. Moreover, they also proved an existence theorem for a strong vector equilibrium problem (1.5) (see [4]).

On the other hand, it is well known that a strong solution of vector equilibrium problem is an ideal solution; it is better than other solutions such as efficient solution, weak efficient solution, proper efficient solution, and supper efficient solution (see [12]). Thus, it is important to study the existence of strong solution and properties of the strong solution set. In 2003, Ansari and Flores-Bazán [26] considered the following generalized vector quasiequilibrium problem (for short, GVQEP): to find $x \in K$ such that

$$
x \in A(x): F(x, y) \not \subset-\operatorname{int} C \quad \forall y \in A(x) .
$$

Very recently, the generalized strong vector quasiequilibrium problem (in short, GSVQEP) is introduced by Hou et al. [27] and Long et al. [16]. Let $X, Y$, and $Z$ be real 
locally convex Hausdorff topological vector spaces, $K \subset X$ and $D \subset Y$ nonempty compact convex subsets, and $C \subset Z$ a nonempty closed convex cone. Let $S: K \rightarrow 2^{K}, T: K \rightarrow 2^{D}$ and $F: K \times D \times K \rightarrow 2^{Z}$ be three set-valued mappings. They considered the GSVQEP, finding $x \in K, y \in T(X)$ such that $x \in S(x)$ and

$$
F(x, y, x) \subset C, \quad \forall x \in S(x) .
$$

Moreover, they gave an existence theorem for a generalized strong vector quasiequilibrium problem without assuming that the dual of the ordering cone has a weak* compact base.

Throughout this paper, motivated and inspired by Hou et al. [27], Long et al. [16], and Yuan [28], we will introduce and study the generalized vector quasiequilibrium problem on locally G-convex Hausdorff topological vector spaces. Let $X, Y$, and $Z$ be real locally $G$ convex Hausdorff topological vector spaces, $K \subset X$ and $D \subset Y$ nonempty compact subsets, and $C \subset Z$ a nonempty closed convex cone. We also suppose that $F: K \times D \times K \rightarrow 2^{Z}$, $S: K \rightarrow 2^{K}$ and $T: K \rightarrow 2^{D}$ are set-valued mappings.

The generalized vector quasiequilibrium problem of type (I) (GSVQEP I) is to find $x^{*} \in K$ and $y^{*} \in T\left(x^{*}\right)$ such that

$$
x^{*} \in S\left(x^{*}\right), \quad F\left(x^{*}, y^{*}, z\right) \subset C \quad \forall z \in S\left(x^{*}\right) .
$$

The generalized vector quasiequilibrium problem of type (II) (GSVQEP II) is to find $x^{*} \in K$ and $y^{*} \in T\left(x^{*}\right)$ such that

$$
x^{*} \in S\left(x^{*}\right), \quad F\left(x^{*}, y^{*}, z\right) \not \subset C \quad \forall z \in S\left(x^{*}\right) .
$$

We denote the set of all solution to the (GSVQEP I) and (GSVQEP II) by $V_{s}(F)$ and $V_{w}(F)$, respectively. The main motivation of this paper is to prove the existence theorems of the generalized strong vector quasiequilibrium problems in locally G-convex spaces, by using Kakutani-Fan-Glicksberg fixed point theorem for upper semicontinuous set-valued mapping with nonempty closed acyclic values, and the closedness of $V_{s}(F)$ and $V_{w}(F)$. The results in this paper generalize, extend, and unify some well-known some existence theorems in the literature.

\section{Preliminaries}

Let $\Delta_{n}$ be the standard $n$-dimensional simplex in $R^{n+1}$ with vertices $e_{0}, e_{1}, e_{2}, \ldots, e_{n}$. For any nonempty subset $J$ of $\{0,1,2, \ldots, n\}$, we denote $\Delta_{J}$ by the convex hull of the vertices $\left\{e_{j}: j \in\right.$ $J\}$. The following definition was essentially given by Park and Kim [29].

Definition 2.1. A generalised convex space, or say, a $G$-convex space $(X, D, \Gamma)$ consists of a topological space $X$, a nonempty subset $D$ of $X$ and a function $\Gamma: \mathcal{F} \rightarrow X \backslash\{\emptyset\}$ such that

(i) for each $A, B \in \mathcal{F}(X), \Gamma(A) \subset \Gamma(B)$ if $A \subset B$,

(ii) for each $A \in \mathcal{F}(X)$ with $|A|=n+1$, there exists a continuous function $\phi_{A}$ : $\Delta_{n} \rightarrow \Gamma(A)$ such that $\phi_{A}\left(\Delta_{J}\right) \subset \Gamma(J)$ for each $\emptyset \neq J \subset\{0,1,2, \ldots, n\}$, where $A=\left\{x_{0}, x_{1}, x_{2}, \ldots, x_{n}\right\}$ and $\Delta_{J}$ denotes the face of $\Delta_{n}$ corresponding to the subindex of $J$ in $\{0,1,2, \ldots, n\}$. 
A subset $C$ of the $G$-convex space $(X, D, \Gamma)$ is said to be $G$-convex if for each $A \in$ $F(D), \Gamma_{A} \subset C$ for all $A \subset C$. For the convenience of our discussion, we also denote $\Gamma(A)$ by $\Gamma_{A}$ or $\Gamma_{N}$ if there is no confusion for $A=\left\{x_{0}, x_{1}, x_{2}, \ldots, x_{n}\right\} \in \mathcal{F}(X)$, where $N$ is the set of all indices for the set $A$; that is, $N=\{0,1,2, \ldots, n\}$. A space $X$ is said to have a $G$-convex structure if and only if $X$ is a $G$-convex space.

In order to cover general economic models without linear convex structures, Park and Kim [29] introduced another abstract convexity notion called a G-convex space, which includes many abstract convexity notions such as $H$-convex spaces as special cases. For the details on G-convex spaces, see [30-34], where basic theory was extensively developed.

Definition 2.2. A G-convex $X$ is said to be a locally $G$-convex space if $X$ is a uniform topological space with uniformity $\mathcal{U}$, which has an open base $\mathbb{B}:=\left\{V_{i}: i \in I\right\}$ of symmetric entourages such that for each $V \in \mathbb{B}$, the set $V(x):=\{y \in X:(y, x) \in V\}$ is a $G$-convex set for each $x \in X$.

We recall that a nonempty space is said to be acyclic if all of its reduced Čech homology groups over the rationals vanish.

Definition 2.3 (see [35]). Let $E$ be a topological space. A subset $D$ of $E$ is called contractible at $v \in D$, if there is a continuous mapping $F: D \times[0,1] \rightarrow D$ such that $F(u, 0)=u$ for all $u \in D$ and $F(u, 1)=v$ for all $u \in D$.

In particular, each contractible space is acyclic and thus any nonempty convex or starshaped set is acyclic. Moreover, by the definition of contractible set, we see that each convex space is contractible.

Definition 2.4. Let $X$ and $Y$ be two topological vector spaces and $K$ a nonempty subset of $X$, and let $F: K \rightarrow 2^{Y}$ be a set-valued mapping.

(i) $F$ is called upper $C$-continuous at $x_{0} \in K$ if, for any neighbourhood $U$ of the origin in $Y$, there is a neighbourhood $V$ of $x_{0}$ such that, for all $x \in V$,

$$
F(x) \subset F\left(x_{0}\right)+U+C
$$

(ii) $F$ is called lower $C$-continuous at $x_{0} \in K$ if, for any neighbourhood $U$ of the origin in $Y$, there is a neighbourhood $V$ of $x_{0}$ such that for all $x \in V$,

$$
F\left(x_{0}\right) \subset F(x)+U-C .
$$

Definition 2.5. Let $X$ and $Y$ be two topological vector spaces and $K$ a nonempty convex subset of $X$. A set-valued mapping $F: K \rightarrow 2^{Y}$ is said to be properly $C$-quasiconvex if, for any $x, y \in K$ and $t \in[0,1]$, we have

$$
\text { either } F(x) \subset F(t x+(1-t) y)+C \text { or } F(y) \subset F(t x+(1-t) y)+C
$$

Definition 2.6. Let $X$ and $Y$ be two topological vector spaces and $T: X \rightarrow 2^{Y}$ a set-valued mapping. 
(i) $T$ is said to be upper semicontinuous at $x \in X$ if, for any open set $V$ containing $T(x)$, there exists an open set $U$ containing $x$ such that for all $t \in U, T(t) \subset V$; $T$ is said to be upper semicontinuous on $X$ if it is upper semicontinuous at all $x \in X$.

(ii) $T$ is said to be lower semicontinuous at $x \in X$ if, for any open set $V$ with $T(x) \cap V \neq \emptyset$, there exists an open set $U$ containing $x$ such that for all $t \in U, T(t) \cap V \neq \emptyset ; T$ is said to be lower semicontinuous on $\mathrm{X}$ if it is lower semicontinuous at all $x \in \mathrm{X}$.

(iii) $T$ is said to be continuous on $X$ if it is at the same time upper semicontinuous and lower semicontinuous on $X$.

(iv) $T$ is said to be closed if the graph, $\operatorname{Graph}(T)$, of $T$, that is, $\operatorname{Graph}(T)=\{(x, y): x \in$ $X$ and $y \in T(x)\}$, is a closed set in $X \times Y$.

Lemma 2.7 (see [36]). Let $X$ and $Y$ be two Hausdorff topological vector spaces and $T: X \rightarrow 2^{Y}$ a set-valued mapping. Then, the following properties hold:

(i) if $T$ is closed and $\overline{T(X)}$ is compact, then $T$ is upper semicontinuous, where $T(X)=$ $\cup_{x \in X} T(x)$ and $\bar{E}$ denotes the closure of the set $E$,

(ii) if $T$ is upper semicontinuous and for any $x \in X, T(x)$ is closed, then $T$ is closed,

(iii) $T$ is lower semicontinuous at $x \in X$ if and only if for any $y \in T(x)$ and any net $\left\{x_{\alpha}\right\}, x_{\alpha} \rightarrow$ $x$, there exists a net $\left\{y_{\alpha}\right\}$ such that $y_{\alpha} \in T\left(x_{\alpha}\right)$ and $y_{\alpha} \rightarrow y$.

We now have the following fixed point theorem in locally G-convex spaces given by Yuan [28] which is a generalization of the Fan-Glickberg-type fixed point theorems for upper semicontinuous set-valued mapping with nonempty closed acyclic values given in several places (e.g., see Kirk and Shin [37], Park and Kim [29], and others in locally convex spaces).

Lemma 2.8 (see [28]). Let $X$ be a compact locally G-convex space and $F: X \rightarrow 2^{X}$ an upper semicontinuous set-valued mappings with nonempty closed acyclic values. Then, $F$ has a fixed point; that is, there exists an $x^{*} \in X$ such that $x^{*} \in F\left(x^{*}\right)$.

\section{Main Results}

In this section, we apply the Kakutani-Fan-Glicksberg fixed point theorem for upper semicontinuous set-valued mapping with nonempty closed acyclic values to establish two existence theorems of strong solutions and obtain the closedness of the strong solutions set for generalized strong vector quasiequilibrium problem.

Theorem 3.1. Let $X, Y$, and $Z$ be real locally G-convex topological vector spaces, $K \subset X$ and $D \subset Y$ nonempty compact subsets, and $C \subset Z$ a nonempty closed convex cone. Let $S: K \rightarrow 2^{K}$ be a continuous set-valued mapping such that for any $x \in K$, the set $S(x)$ is a nonempty closed contractible subset of $K$. Let $T: K \rightarrow 2^{D}$ be an upper semicontinuous set-valued mapping with nonempty closed acyclic values and $F: K \times D \times K \rightarrow 2^{Z}$ a set-valued mapping satisfy the following conditions:

(i) for all $(x, y) \in K \times D, F(x, y, S(x)) \subset C$,

(ii) for all $(y, z) \in D \times K, F(\cdot, y, z)$ are properly $C$-quasiconvex,

(iii) $F(\cdot, \cdot, \cdot)$ are upper $C$-continuous,

(iv) for all $y \in D, F(\cdot, y, \cdot)$ are lower $(-C)$-continuous.

Then, the solutions set $V_{S}(F)$ is nonempty and closed subset of $K$. 
Proof. For any $(x, y) \in K \times D$, we define a set-valued mapping $G: K \times D \rightarrow 2^{K}$ by

$$
G(x, y)=\{u \in S(x): F(u, y, z) \subset C, \forall z \in S(x)\}
$$

Since for any $(x, y) \in K \times D, S(x)$ is nonempty. So, by assumption (i), we have that $G(x, y)$ is nonempty. Next, we divide the proof into five steps.

Step 1 (to show that $G(x, y)$ is acyclic). Since every contractible set is acyclic, it is enough to show that $G(x, y)$ is contractible. Let $u \in G(x, y)$, thus $u \in S(x)$ and $F(u, y, z) \subset C$ for all $z \in$ $S(x)$. Since $S(x)$ is contractible, there exists a continuous mapping $h: S(x) \times[0,1] \rightarrow S(x)$ such that $h(s, 0)=s$ for all $s \in S(x)$ and $h(s, 1)=u$ for all $s \in S(x)$. Now, we set $H(s, t)=$ $t u+(1-t) h(s, t)$ for all $(s, t) \in G(x, y) \times[0,1]$. Then, $H$ is a continuous mapping, and we see that $H(s, 0)=s$ for all $s \in G(x, y)$ and $H(s, 1)=u$ for all $s \in G(x, y)$. Let $(s, t) \in G(x, y) \times[0,1]$. We claim that $H(s, t) \in G(x, y)$. In fact, if $H(s, t) \notin G(x, y)$, then there exists $z^{*} \in S(x)$ such that

$$
F\left(H(s, t), y, z^{*}\right) \not \subset C \text {. }
$$

Since $\left(\cdot, y, z^{*}\right)$ is properly $C$-quasiconvex, we can assume that

$$
F\left(u, y, z^{*}\right) \subset F\left(t u+(1-t) h(s, t), y, z^{*}\right)+C .
$$

It follows that

$$
F\left(u, y, z^{*}\right) \subset F\left(H(s, t), y, z^{*}\right)+C \not \subset C+C \subset C,
$$

which contradicts $u \in G(x, y)$. Therefore, $H(s, t) \in G(x, y)$, and hence $G(x, y)$ is contractible.

Step 2 (to show that $G(x, y)$ is a closed subset of $K$ ). Let $\left\{a_{\alpha}\right\}$ be a sequence in $G(x, y)$ such that $a_{\alpha} \rightarrow a^{*}$. Then, $a_{\alpha} \in S(x)$. Since $S(x)$ is a closed subset of $K, a^{*} \in S(x)$. Since $S$ is a lower semicontinuous, it follows by Lemma 2.7(iii) that for any $z^{*} \in S(x)$ and any net $\left\{x_{\alpha}\right\} \rightarrow x$, there exists a net $\left\{z_{\alpha}\right\}$ such that $z_{\alpha} \in S\left(x_{\alpha}\right)$ and $z_{\alpha} \rightarrow z^{*}$. This implies that

$$
F\left(a_{\alpha}, y, z_{\alpha}\right) \subset C
$$

Since $F(\cdot, y, \cdot)$ are lower $(-C)$-continuous, we note that for any neighbourhood $U$ of the origin in $Z$, there exists a subnet $\left\{a_{\beta}, z_{\beta}\right\}$ of $\left\{a_{\alpha}, z_{\alpha}\right\}$ such that

$$
F\left(a^{*}, y, z^{*}\right) \subset F\left(a_{\beta}, y, z_{\beta}\right)+U+C .
$$

From (3.5) and (3.6), we have

$$
F\left(a^{*}, y, z^{*}\right) \subset U+C
$$


We claim that $F\left(a^{*}, y, z^{*}\right) \subset C$. Assume that there exists $p \in F\left(a^{*}, y, z^{*}\right)$ and $p \notin C$. Then, we note that $0 \notin(C-p)$, and the set $C-p$ is closed. Thus, $Z \backslash(C-p)$ is open, and $0 \in Z \backslash(C-p)$. Since $Z$ is a locally $G$-convex space, there exists a neighbourhood $U_{0}$ of the origin such that $U_{0} \subset Z \backslash(C-p)$ and $U_{0}=-U_{0}$. Thus, we note that $0 \notin U_{0}+(C-p)$, and hence $p \notin U_{0}+C$, which contradicts to (3.7). Hence, $F\left(a^{*}, y, z^{*}\right) \subset C$, and therefore, $a^{*} \in G(x, y)$. Then, $G(x, y)$ is a closed subset of $K$.

Step 3 (to show that $G(x, y)$ is upper semicontinuous). Let $\left\{\left(x_{\alpha}, y_{\alpha}\right): \alpha \in I\right\} \subset K \times D$ be given such that $\left(x_{\alpha}, y_{\alpha}\right) \rightarrow(x, y) \in K \times D$, and let $a_{\alpha} \in G\left(x_{\alpha}, y_{\alpha}\right)$ such that $a_{\alpha} \rightarrow a$. Since $a_{\alpha} \in S\left(x_{\alpha}\right)$ and $S$ is upper semicontinuous, it follows by Lemma 2.7(ii) that $a \in S(x)$. We claim that $a \in G(x, y)$. Assume that $a \notin G(x, y)$. Then, there exists $z^{*} \in S(x)$ such that

$$
F\left(a, y, z^{*}\right) \not \subset C,
$$

which implies that there is a neighbourhood $U_{0}$ of the origin in $Z$ such that

$$
F\left(a, y, z^{*}\right)+U_{0} \not \subset C
$$

Since $F$ is upper $C$-continuous, it follows that for any neighbourhood $U$ of the origin in $Z$, there exists a neighbourhood $U_{1}$ of $\left(a, y, z^{*}\right)$ such that

$$
F(\widehat{a}, \widehat{y}, \widehat{z}) \subset F\left(a, y, z^{*}\right)+U+C, \quad \forall(\widehat{a}, \widehat{y}, \widehat{z}) \in U_{1}
$$

Without loss of generality, we can assume that $U_{0}=U$. This implies that

$$
F(\widehat{a}, \widehat{y}, \widehat{z}) \subset F\left(a, y, z^{*}\right)+U_{0}+C \not \subset C+C \subset C, \quad \forall(\widehat{a}, \widehat{y}, \widehat{z}) \in U_{1}
$$

Thus, there is $\alpha_{0} \in I$ such that

$$
F\left(a_{\alpha}, y_{\alpha}, z_{\alpha}\right) \not \subset C, \quad \forall \alpha \geq \alpha_{0}
$$

it is a contradiction to $a_{\alpha} \in G\left(x_{\alpha}, y_{\alpha}\right)$. Hence, $a \in G(x, y)$, and therefore, $G$ is a closed mapping. Since $K$ is a compact set and $G(x, y)$ is a closed subset of $K, G(x, y)$ is compact. This implies that $\overline{G(x, y)}$ is compact. Then, by Lemma 2.7(i), we have $G(x, y)$ is upper semicontinuous.

Step 4 (to show that the solutions set $V_{S}(F)$ is nonempty). Define the set-valued mapping $Q: K \times D \rightarrow 2^{K \times D}$ by

$$
Q(x, y)=(G(x, y), T(x)) \quad \forall(x, y) \in K \times D
$$

Then, $Q$ is an upper semicontinuous mpping. Moreover, we note that $Q(x, y)$ is a nonempty closed acyclic subset of $K \times D$ for all $(x, y) \in K \times D$. By Lemma 2.8, there exists a point 
$(\bar{x}, \bar{y}) \in(K \times D)$ such that $(\bar{x}, \bar{y}) \in Q(\bar{x}, \bar{y})$. Thus, we have $\bar{x} \in G(\bar{x}, \bar{y}), \bar{y} \in T(\bar{x})$. It follows that there exists $\bar{x} \in K$ and $\bar{y} \in T(\bar{x})$ such that $\bar{x} \in S(\bar{x})$ and

$$
F(\bar{x}, \bar{y}, z) \subset C \quad \forall z \in S(\bar{x})
$$

Hence, the solutions set $V_{S}(F) \neq \emptyset$.

Step 5 (to show that the solutions set $V_{S}(F)$ is closed). Let $\left\{x_{\alpha}: \alpha \in I\right\}$ be a net in $V_{S}(F)$ such that $x_{\alpha} \rightarrow x^{*}$. By definition of the solutions set $V_{S}(F)$, we note that $x_{\alpha} \in S\left(x_{\alpha}\right)$, and there exist $y_{\alpha} \in T\left(x_{\alpha}\right)$ satisfying

$$
F\left(x_{\alpha}, y_{\alpha}, z\right) \subset C \quad \forall z \in S\left(x_{\alpha}\right)
$$

Since $S$ is a continuous closed valued mapping, $x^{*} \in S\left(x^{*}\right)$. From the compactness of $D$, we can assume that $y_{\alpha} \rightarrow y^{*}$. Since $T$ is an upper semicontinuous closed valued mapping, it follows by Lemma 2.7(ii) that $T$ is closed. Thus, we have $y^{*} \in T\left(x^{*}\right)$. Since $F\left(\cdot, y^{*}, \cdot\right)$ is a lower $(-C)$-continuous, we have

$$
F\left(x^{*}, y^{*}, z\right) \subset C \quad \forall z \in S\left(x^{*}\right)
$$

This means that $x^{*}$ belongs to $V_{S}(F)$. Therefore, the solutions set $V_{S}(F)$ is closed. This completes the proof.

Theorem 3.1 extends Theorem 3.1 of Long et al. [16] to locally G-convex which includes locally convex Hausdorff topological vector spaces.

Corollary 3.2. Let $X, Y$ and $Z$ be real locally convex Hausdorff topological vector spaces, $K \subset X$ and $D \subset Y$ two nonempty compact convex subsets, and $C \subset Z$ a nonempty closed convex cone. Let $S: K \rightarrow 2^{K}$ be a continuous set-valued mapping such that for any $x \in K, S(x)$ is a nonempty closed convex subset of $K$. Let $T: K \rightarrow 2^{D}$ be an upper semicontinuous set-valued mapping such that for any $x \in K, T(x)$ is a nonempty closed convex subset of $D$. Let $F: K \times D \times K \rightarrow 2^{Z}$ be a set-valued mapping satisfying the following conditions:

(i) for all $(x, y) \in K \times D, F(x, y, S(x)) \subset C$,

(ii) for all $(y, z) \in D \times K, F(\cdot, y, z)$ are properly $C$-quasiconvex,

(iii) $F(\cdot, \cdot, \cdot)$ are upper $C$-continuous,

(iv) for all $y \in D, F(\cdot, y, \cdot)$ are lower $(-C)$-continuous.

Then, the solutions set $V_{S}(F)$ is nonempty and closed subset of $K$.

Theorem 3.3. Let $X, Y$ and $Z$ be real locally G-convex topological vector spaces, $K \subset X$ and $D \subset Y$ nonempty compact subsets, and $C \subset Z$ a nonempty closed convex cone. Let $S: K \rightarrow 2^{K}$ be a continuous set-valued mapping such that for any $x \in K$, the set $S(x)$ is a nonempty closed contractible 
subset of $K$. Let $T: K \rightarrow 2^{D}$ be an upper semicontinuous set-valued mapping with nonempty closed acyclic values and $F: K \times D \times K \rightarrow 2^{Z}$ a set-valued mapping satisfying the following conditions:

(i) for all $(x, y) \in K \times D, F(x, y, S(x)) \not \subset C$,

(ii) for all $(y, z) \in D \times K, F(\cdot, y, z)$ are properly $C$-quasiconvex,

(iii) $F(\cdot, \cdot, \cdot)$ are upper $C$-continuous,

(iv) for all $y \in D, F(\cdot, y, \cdot)$ are lower $(-C)$-continuous.

Then, the solutions set $V_{W}(F)$ is nonempty and closed subset of $K$.

Proof. For any $(x, y) \in K \times D$, define a set-valued mapping $B: K \times D \rightarrow 2^{K}$ by

$$
B(x, y)=\{u \in S(x): F(u, y, z) \not \subset C, \forall z \in S(x)\}
$$

Proceeding as in the proof of Theorem 3.1, we need to prove that $B(x, y)$ is closed acyclic subset of $K \times D$ for all $(x, y) \in K \times D$. We divide the remainder of the proof into three steps.

Step 1 (to show that $B(x, y)$ is a closed subset of $K$ ). Let $\left\{a_{\alpha}\right\}$ be a sequence in $B(x, y)$ such that $a_{\alpha} \rightarrow a^{*}$. Then, $a_{\alpha} \in S(x)$ and $F\left(a_{\alpha}, y, z\right) \not \subset C$ for all $z \in S(x)$. Since $S(x)$ is a closed subset of $K$, we have $a^{*} \in S(x)$. By the lower semicontinuity of $S$ and Lemma 2.7(iii), we note that for any $z \in S(x)$ and any net $\left\{x_{\alpha}\right\} \rightarrow x$, there exists a net $\left\{z_{\alpha}\right\}$ such that $z_{\alpha} \in S\left(x_{\alpha}\right)$ and $z_{\alpha} \rightarrow z$. Thus, we have

$$
F\left(a_{\alpha}, y, z_{\alpha}\right) \not \subset C,
$$

which implies that there exists a neighbourhood $U_{0}$ of the origin in $Z$ such that

$$
F\left(a_{\alpha}, y, z_{\alpha}\right)+U_{0} \not \subset C
$$

Since $F(\cdot, y, \cdot)$ are lower $(-C)$-continuous, it follows that for any neighbourhood $U$ of the origin in $Z$, there exists a subnet $\left\{a_{\beta}, z_{\beta}\right\}$ of $\left\{a_{\alpha}, z_{\alpha}\right\}$ such that

$$
F\left(a^{*}, y, z\right) \subset F\left(a_{\beta}, y, z_{\beta}\right)+U+C
$$

Without loss of generality, we can assume that $U=U_{0}$. Then, by (3.18), (3.19), and (3.20), we have

$$
F\left(a^{*}, y, z\right) \subset F\left(a_{\alpha}, y, z_{\alpha}\right)+U_{0}+C \not \subset C+C \subset C .
$$

This means that $a^{*} \in B(x, y)$ and so $B(x, y)$ is a closed subset of $K$.

Step 2 (to show that $B(x, y)$ is upper semicontinuous). Let $\left\{\left(x_{\alpha}, y_{\alpha}\right): \alpha \in I\right\} \subset K \times D$ be given such that $\left(x_{\alpha}, y_{\alpha}\right) \rightarrow(x, y) \in K \times D$, and let $a_{\alpha} \in B\left(x_{\alpha}, y_{\alpha}\right)$ such that $a_{\alpha} \rightarrow a$. Then, $a_{\alpha} \in$ $S\left(x_{\alpha}\right)$ and $F\left(a_{\alpha}, y, z\right) \not \subset C$, for all $z \in S\left(x_{\alpha}\right)$. Since $S$ is upper semicontinuous closed valued 
mapping, it follows by Lemma 2.7(ii) that $a \in S(x)$. We claim that $a \in B(x, y)$. Indeed, if $a \notin B(x, y)$, then there exists a $z_{0} \in S(x)$ such that

$$
F\left(a, y, z_{0}\right) \subset \mathrm{C} .
$$

Since $F$ is upper $C$-continuous, we note that for any neighbourhood $U$ of the origin in $Z$, there exists a neighbourhood $U_{0}$ of $\left(a, y, z_{0}\right)$ such that

$$
F\left(a^{*}, y^{*}, z^{*}\right) \subset F\left(a, y, z_{0}\right)+U+C, \quad \forall\left(a^{*}, y^{*}, z^{*}\right) \in U_{0}
$$

From (3.22) and (3.23), we obtain

$$
F\left(a^{*}, y^{*}, z^{*}\right) \subset U+C, \quad \forall\left(a^{*}, y^{*}, z^{*}\right) \in U_{0} .
$$

As in the proof of Step 2 in Theorem 3.1, we can show that $F\left(a^{*}, y^{*}, z^{*}\right) \subset C$ for all $\left(a^{*}, y^{*}, z^{*}\right) \in$ $U_{0}$. Hence, there is $\alpha_{0} \in I$ such that

$$
F\left(a_{\alpha}, y_{\alpha}, z_{\alpha}\right) \subset C, \quad \forall \alpha \geq \alpha_{0}
$$

it is a contradiction to $a_{\alpha} \in B\left(x_{\alpha}, y_{\alpha}\right)$. Hence, $a \in B(x, y)$, and therefore, $B$ is a closed mapping. Since $K$ is a compact set and $B(x, y)$ is a closed subset of $K, B(x, y)$ is compact. This implies that $\overline{B(x, y)}$ is compact. Then, by Lemma 2.7(i), we have that $B(x, y)$ is upper semicontinuous.

Step 3 (to show that the solutions set $V_{W}(F)$ is nonempty and closed). Define the set-valued mapping $P: K \times D \rightarrow 2^{K \times D}$ by

$$
P(x, y)=(B(x, y), T(x)) \quad \forall(x, y) \in K \times D .
$$

Then, $P$ is an upper semicontinuous mapping. Moreover, we note that $P(x, y)$ is a nonempty closed acyclic subset of $K \times D$ for all $(x, y) \in K \times D$. Hence, by Lemma 2.8, there exists a point $(\bar{x}, \bar{y}) \in(K \times D)$ such that $(\bar{x}, \bar{y}) \in P(\bar{x}, \bar{y})$. Thus, we have $\bar{x} \in B(\bar{x}, \bar{y})$ and $\bar{y} \in T(\bar{x})$. This implies that there exists $\bar{x} \in K$ and $\bar{y} \in T(\bar{x})$ such that $\bar{x} \in S(\bar{x})$ and

$$
F(\bar{x}, \bar{y}, z) \not \subset C \quad \forall z \in S(\bar{x})
$$

Hence, $V_{W}(F) \neq \emptyset$. Similarly, by the proof of Step 5 in Theorem 3.1, we have $V_{W}(F)$ is closed. This completes the proof.

\section{Stability}

In this section, we discuss the stability of the solutions for the generalized strong vector quasiequilibrium problem (GSVQEP II).

Throughout this section, let $X, Y$ be Banach spaces, and let $Z$ be a real locally $G$-convex Hausdorff topological vector space. Let $K \subset X$ and $D \subset Y$ be nonempty compact subsets, and 
let $C \subset Z$ be a nonempty closed convex cone. Let $E:=\left\{(S, T) \mid S: K \rightarrow 2^{K}\right.$ is a continuous set-valued mapping with nonempty closed contractible values, and $T: K \rightarrow 2^{D}$ is an upper semicontinuous set-valued mapping with nonempty closed acyclic values\}. defined by

Let $B_{1}, B_{2}$ be compact sets in a normed space. Recall that the Hausdorff metric is

$$
H\left(B_{1}, B_{2}\right):=\max \left\{\sup _{b \in B_{1}} d\left(b, B_{2}\right), \sup _{b \in B_{2}} d\left(b, B_{1}\right)\right\},
$$

where $d\left(b, B_{2}\right):=\inf _{a \in B_{2}}\|b-a\|$.

For $\left(S_{1}, T_{1}\right),\left(S_{2}, T_{2}\right) \in E$, we define

$$
\rho\left(\left(S_{1}, T_{1}\right),\left(S_{2}, T_{2}\right)\right):=\sup _{x \in K} H_{1}\left(S_{1}(x), S_{2}(x)\right)+\sup _{x \in K} H_{2}\left(T_{1}(x), T_{2}(x)\right),
$$

where $H_{1}, H_{2}$ being the appropriate Hausdorff metrics. Obviously, $(E, \rho)$ is a metric space. Now, we assume that $F$ satisfies the assumptions of Theorem 3.3. Then, for each $(S, T) \in E$, (GSVQEP II) has a solution $x^{*}$. Let

$$
\varphi(S, T)=\{x \in K: x \in S(x), \exists y \in T(x), F(x, y, z) \not \subset C \forall z \in S(x)\}
$$

Thus, $\varphi(S, T) \neq \emptyset$, which conclude that $\varphi$ defines a set-valued mapping from $E$ into $K$.

We also need the following lemma in the sequel.

Lemma 4.1 (see $[8,38])$. Let $W$ be a metric space, and let $A, A_{n}(n=1,2, \ldots)$ be compact sets in $W$. Suppose that for any open set $O \supset A$, there exists $n_{0}$ such that $A_{n} \supset O$ for all $n \geq n_{0}$. Then, any sequence $\left\{x_{n}\right\}$ satisfying $x_{n} \in A_{n}$ has a convergent subsequence with limit in $A$.

In the following theorem, we replaced the convex set by the contractible set and acyclic set in Theorem 4.1 in [16]. The following theorem can acquire the same result appearing on the Theorem 4.1 by utilized Lemma 4.1. Now, we need only to present stability theorem for the solution set mapping $\varphi$ for (GSVQEP II).

Theorem 4.2. $\varphi: E \rightarrow 2^{K}$ is an upper semicontinuous mapping with compact values.

Proof. Since $K$ is compact, we need only to show that $\varphi$ is a closed mapping. In fact, let $\left(\left(S_{n}, T_{n}\right), x_{n}\right) \in \operatorname{Graph}(\varphi)$ be such that $\left(\left(S_{n}, T_{n}\right), x_{n}\right) \rightarrow\left((S, T), x^{*}\right)$. Since $x_{n} \in \varphi\left(S_{n}, T_{n}\right)$, we have $x_{n} \in S_{n}\left(x_{n}\right)$, and there exists $y_{n} \in T_{n}\left(x_{n}\right)$ such that

$$
F\left(x_{n}, y_{n}, z\right) \not \subset C \quad \forall z \in S_{n}\left(x_{n}\right)
$$

By the same argument as in the proof of Theorem 4.1 in [16], we can show that $x^{*} \in S\left(x^{*}\right)$ and $y^{*} \in T\left(x^{*}\right)$.

Since $S$ is lower semicontinuous at $x^{*}$ and $x_{n} \rightarrow x^{*}$, it follows by Lemma 2.7(iii) that for any $z \in S\left(x^{*}\right)$, there exists $z_{n} \in S\left(x_{n}\right)$ such that $z_{n} \rightarrow z$. To finish the proof of the theorem, we need to show that $F\left(x^{*}, y^{*}, z\right) \not \subset C$ for all $z \in S\left(x^{*}\right)$. Since $\rho\left(\left(S_{n}, T_{n}\right),(S, T)\right) \rightarrow 0$, it follows 
by the same argument as in the proof of Theorem 4.1 in [16] that there exists a subsequence $\left\{x_{n_{k}}\right\}$ of $\left\{x_{n}\right\}$ such that $x_{n_{k}} \in S_{n_{k}}\left(x_{n_{k}}\right), y_{n_{k}} \in T_{n_{k}}\left(x_{n_{k}}\right), z_{n_{k}} \in S_{n_{k}}\left(x_{n_{k}}\right)$, and

$$
F\left(x_{n_{k}}, y_{n_{k}}, z_{n_{k}}\right) \not \subset C \text {. }
$$

From the upper $C$-continuous of $F$, we have

$$
F\left(x^{*}, y^{*}, z\right) \not \subset C \quad \forall z \in S\left(x^{*}\right) \text {. }
$$

Then, $\left((S, T), x^{*}\right) \in \operatorname{Graph}(\varphi)$, and so $\operatorname{Graph}(\varphi)$ is closed. The theorem is proved.

\section{Acknowledgments}

S. Plubtieng would like to thank the Thailand Research Fund for financial support under Grant no. BRG5280016. Moreover, K. Sitthithakerngkiet would like to thank the Office of the Higher Education Commission, Thailand, for supporting by grant fund under Grant no. CHE-Ph.D-SW-RG/41/2550, Thailand.

\section{References}

[1] E. Blum and W. Oettli, "From optimization and variational inequalities to equilibrium problems," The Mathematics Student, vol. 63, no. 1-4, pp. 123-145, 1994.

[2] M. Bianchi and S. Schaible, "Generalized monotone bifunctions and equilibrium problems," Journal of Optimization Theory and Applications, vol. 90, no. 1, pp. 31-43, 1996.

[3] W. Oettli and D. Schläger, "Existence of equilibria for monotone multivalued mappings," Mathematical Methods of Operations Research, vol. 48, no. 2, pp. 219-228, 1998.

[4] Q. H. Ansari, W. Oettli, and D. Schläger, "A generalization of vectorial equilibria," Mathematical Methods of Operations Research, vol. 46, no. 2, pp. 147-152, 1997.

[5] M. Bianchi, N. Hadjisavvas, and S. Schaible, "Vector equilibrium problems with generalized monotone bifunctions," Journal of Optimization Theory and Applications, vol. 92, no. 3, pp. 527-542, 1997.

[6] G. Debreu, "A social equilibrium existence theorem," Proceedings of the National Academy of Sciences of the United States of America, vol. 38, pp. 886-893, 1952.

[7] J.-Y. Fu, "Generalized vector quasi-equilibrium problems," Mathematical Methods of Operations Research, vol. 52, no. 1, pp. 57-64, 2000.

[8] F. Gianness, Ed., Vector Variational Inequalities and Vector Equilibria. Mathematical Theories, vol. 38 of Nonconvex Optimization and Its Applications, Kluwer Academic Publishers, Dordrecht, The Netherlands, 2000.

[9] X. Gong, "Strong vector equilibrium problems," Journal of Global Optimization, vol. 36, no. 3, pp. 339$349,2006$.

[10] X. H. Gong, “Efficiency and Henig efficiency for vector equilibrium problems," Journal of Optimization Theory and Applications, vol. 108, no. 1, pp. 139-154, 2001.

[11] R. B. Holmes, Geometric Functional Analysis and Its Applications, Springer, New York, NY, USA, 1975 Graduate Texts in Mathematics, no. 2.

[12] S. H. Hou, X. H. Gong, and X. M. Yang, "Existence and stability of solutions for generalized Ky fan inequality problems with trifunctions," Journal of Optimization Theory and Applications, vol. 146, no. 2, pp. 387-398, 2010.

[13] N. J. Huang, J. Li, and H. B. Thompson, "Stability for parametric implicit vector equilibrium problems," Mathematical and Computer Modelling, vol. 43, no. 11-12, pp. 1267-1274, 2006.

[14] S. J. Li, K. L. Teo, and X. Q. Yang, "Generalized vector quasi-equilibrium problems," Mathematical Methods of Operations Research, vol. 61, no. 3, pp. 385-397, 2005. 
[15] L.-J. Lin and S. Park, “On some generalized quasi-equilibrium problems," Journal of Mathematical Analysis and Applications, vol. 224, no. 2, pp. 167-181, 1998.

[16] X.-J. Long, N.-J. Huang, and K.-1. Teo, "Existence and stability of solutions for generalized strong vector quasi-equilibrium problem," Mathematical and Computer Modelling, vol. 47, no. 3-4, pp. 445451, 2008.

[17] Q. H. Ansari, "Vector equilibrium problems and vector variational inequalities," in Vector Variational Inequalities and Vector Equilibria, F. Giannessi, Ed., vol. 38 of Nonconvex Optim. Appl., pp. 1-15, Kluwer Academic Publishers, Dordrecht, The Netherlands, 2000.

[18] N. X. Tan and P. N. Tinh, "On the existence of equilibrium points of vector functions," Numerical Functional Analysis and Optimization, vol. 19, no. 1-2, pp. 141-156, 1998.

[19] Q. H. Ansari and J.-C. Yao, "On vector quasi-equilibrium problems," in Equilibrium Problems and Variational Models (Erice, 2000), A. Maugeri and F. Giannessi, Eds., vol. 68 of Nonconvex Optim. Appl., pp. 1-18, Kluwer Academic Publishers, Norwell, Mass, USA, 2003.

[20] Q. H. Ansari, I. V. Konnov, and J. C. Yao, “On generalized vector equilibrium problems," Nonlinear Analysis: Theory, Methods \& Applications, vol. 47, no. 1, pp. 543-554, 2001.

[21] Q. H. Ansari and J.-C. Yao, "An existence result for the generalized vector equilibrium problem," Applied Mathematics Letters, vol. 12, no. 8, pp. 53-56, 1999.

[22] I. V. Konnov and J. C. Yao, "Existence of solutions for generalized vector equilibrium problems," Journal of Mathematical Analysis and Applications, vol. 233, no. 1, pp. 328-335, 1999.

[23] Q. H. Ansari, A. H. Siddiqi, and S. Y. Wu, "Existence and duality of generalized vector equilibrium problems," Journal of Mathematical Analysis and Applications, vol. 259, no. 1, pp. 115-126, 2001.

[24] P. Gr. Georgiev and T. Tanaka, "Vector-valued set-valued variants of Ky Fan's inequality," Journal of Nonlinear and Convex Analysis, vol. 1, no. 3, pp. 245-254, 2000.

[25] W. Song, "Vector equilibrium problems with set-valued mappings," in Vector Variational Inequalities and Vector Equilibria, F. Giannessi, Ed., vol. 38 of Nonconvex Optim. Appl., pp. 403-422, Kluwer Academic Publishers, Dordrecht, The Netherlands, 2000.

[26] Q. H. Ansari and F. Flores-Bazán, “Generalized vector quasi-equilibrium problems with applications," Journal of Mathematical Analysis and Applications, vol. 277, no. 1, pp. 246-256, 2003.

[27] S. H. Hou, H. Yu, and G. Y. Chen, "On vector quasi-equilibrium problems with set-valued maps," Journal of Optimization Theory and Applications, vol. 119, no. 3, pp. 485-498, 2003.

[28] G. X.-Z. Yuan, "Fixed points of upper semicontinuous mappings in locally G-convex spaces," Bulletin of the Australian Mathematical Society, vol. 58, no. 3, pp. 469-478, 1998.

[29] S. Park and H. Kim, "Admissible classes of multifunctions on generalized convex spaces," Proceedings of the College of Natural Sciences, vol. 18, pp. 1-21, 1993.

[30] S. Park, "Fixed points of better admissible maps on generalized convex spaces," Journal of the Korean Mathematical Society, vol. 37, no. 6, pp. 885-899, 2000.

[31] S. Park, "New topological versions of the Fan-Browder fixed point theorem," Nonlinear Analysis: Theory, Methods E Applications, vol. 47, no. 1, pp. 595-606, 2001.

[32] S. Park, "Fixed point theorems in locally G-convex spaces," Nonlinear Analysis: Theory, Methods $\mathcal{E}$ Applications, vol. 48, no. 6, pp. 869-879, 2002.

[33] S. Park, "Remarks on acyclic versions of generalized von Neumann and Nash equilibrium theorems," Applied Mathematics Letters, vol. 15, no. 5, pp. 641-647, 2002.

[34] S. Park, "Fixed points of approximable or Kakutani maps in generalized convex spaces," Journal of Nonlinear and Convex Analysis, vol. 7, no. 1, pp. 1-17, 2006.

[35] C. Bardaro and R. Ceppitelli, "Some further generalizations of Knaster-Kuratowski-Mazurkiewicz theorem and minimax inequalities," Journal of Mathematical Analysis and Applications, vol. 132, no. 2, pp. 484-490, 1988.

[36] J.-P. Aubin and I. Ekeland, Applied Nonlinear Analysis, Pure and Applied Mathematics (New York), John Wiley \& Sons, New York, NY, USA, 1984.

[37] W. A. Kirk and S. S. Shin, "Fixed point theorems in hyperconvex spaces," Houston Journal of Mathematics, vol. 23, no. 1, pp. 175-188, 1997.

[38] J. Yu, "Essential weak efficient solution in multiobjective optimization problems," Journal of Mathematical Analysis and Applications, vol. 166, no. 1, pp. 230-235, 1992. 